\title{
Input-delay model predictive control of inland waterways considering the backwater effect
}

\author{
P. Segovia ${ }^{1,2,3}$, L. Rajaoarisoa ${ }^{3}$, F. Nejjari ${ }^{1}$, E. Duviella ${ }^{3}$, V. Puig ${ }^{1,2}$
}

\begin{abstract}
Inland waterways are large-scale systems, generally characterized by negligible bottom slopes and large time delays. These features pose challenging problems at the modeling and controller design stages. A control-oriented model is derived in this work, which allows to handle these issues in a suitable manner. A predictive control scheme is developed to ensure the coordination of the control actions and their delayed effects in the system. The proposed approach is tested on a case study to highlight its performance, and it is shown that it is possible to guarantee the navigability condition of the waterways as well as other operational goals.
\end{abstract}

\section{INTRODUCTION}

Inland waterways transport has flourished in the last years as it offers several interesting economical and environmental benefits over road transport and constitutes a safer way of transporting goods [1]. For these reasons, a progressive increase in the navigation demand is expected in the near future. Furthermore, the current climate change context, which is expected to aggravate [2], will contribute to constrain severely the management of these systems.

Inland waterways management regards the allocation of the available water resources to meet the objectives, being the most important one to keep the water levels within a predefined interval that guarantees the navigability regardless of the weather condition. In order to understand and control the system, its model is needed. However, this is not an easy task in the case of inland waterways, since they are often characterized by a large spatial dimensionality, nonlinear dynamics and rather large time delays. A common approach to this problem consists in partitioning these systems into reaches and modeling each reach separately. A reach is defined as the portion of the water current within two controlled hydraulic structures (e.g. gates and weirs). The final system model is built up by aggregating the models of the reaches while taking into account the network topology.

Water resources management has gained a lot of interest in the last years. Optimal control techniques have been considered to find satisfactory solutions to this problem. One of the most accepted techniques for complex, largescale systems is Model Predictive Control (MPC). As its name implies, the set of controlled inputs is computed ahead

\footnotetext{
${ }^{1}$ P. Segovia, F. Nejjari and V. Puig are with the Research Center for Supervision, Safety and Automatic Control (CS2AC), Universitat Politècnica de Catalunya (UPC), Terrassa Campus, Gaia Building, Rambla Sant Nebridi 22, 08222 Terrassa, Spain.

${ }^{2} \mathrm{P}$. Segovia and V. Puig are with Institut de Robòtica i Informàtica Industrial (CSIC-UPC), c/ Llorens i Artigas 4-6, 08028 Barcelona, Spain.

${ }^{3}$ P. Segovia, L. Rajaoarisoa and E. Duviella are with IMT Lille Douai, Univ. Lille, Unité de Recherche Informatique Automatique, Lille, France.

${ }^{*}$ Corresponding author: P. Segovia: pablo.segovia@upc. edu
}

of time to achieve the optimal performance of the system (represented by the model) regarding the control objectives while respecting the operational limits.

Many works within the framework of MPC for open-flow water systems can be found in the literature. A centralized predictive control approach was presented and tested in a two-pool system by using the SIC $2^{1}$ software in [3]. The same software tool was used in [4] to build a distributed MPC for an irrigation canal. A multivariable predictive controller was developed and implemented in real time in [5], aiming at regulating the downstream water levels of a multi-pool irrigation canal prototype. A coordination between several distributed MPC schemes in order to fulfill the national management objectives was carried out in [6]. A hierarchical distributed MPC approach applied to irrigation canal planning was presented in [7], with a risk management strategy at the highest level. A methodology for the optimal management of combined water supply and navigability in river systems based on MPC was detailed in [8]. Another MPC approach linking transport of and transport over water was presented in [9] and tested in a navigation reach that belongs to the inland waterways network in the north of France. An extended subsystem of the same network, which featured a reach with a distributary, was considered in a decentralized predictive scheme in [10].

\section{Summary of the paper and contribution}

This work regards the development of an MPC approach dedicated to inland waterways in order to guarantee the navigability condition while fulfilling other operational goals. A two-level control framework is considered: the high-level controller (represented by the MPC controller) dictates the setpoints to the local controllers available at each actuator. In turn, these local controllers must ensure that the adequate flows are supplied through the actuators. However, only the high-level control layer is dealt with.

The main contributions of the present work are:

- In many applications in this framework (e.g. irrigation and drainage canals, sewage systems, etc.), when the water waves impact upon the downstream hydraulic structure (backwater effect), they do not flow in the opposite direction due to the non-negligible slope of the reaches. However, navigation canals are usually characterized by null bottom slope, which allows this bidirectional flow. The back-and-forth mass transport,

\footnotetext{
${ }^{1}$ http://sic.g-eau.net/
} 
known as the resonance phenomena, is explicitly taken into account by controlling the upstream water level.

- The transport delays due to the large dimensions of the network constitute a challenging issue to be handled. The coordination between the actual control actions and their delayed effect must be ensured at the controller design stage. This task is simplified thanks to the control-oriented model formulation derived in this work, which allows to handle this problem in an easier and more straightforward manner.

The rest of the paper is organized as follows: Section II presents the well-known control-oriented Integrator Delay Zero (IDZ) model and an equivalent state-space formulation is obtained, aiming at addressing more conveniently the aforementioned challenges. The system constraints, the operational goals and the control design are discussed in Section III. This methodology is illustrated by means of a case study in Section IV, where results are shown and the performance of the controller is discussed. Finally, conclusions are drawn and future steps are outlined in Section V.

\section{Notation}

Throughout this paper, let $\mathbb{R}^{n}$ denote the set of column real vectors of length $n$. Scalars are denoted with either lowercase or uppercase letters (e.g. $\alpha, a, A$, etc.); vectors, with bold lowercase letters (e.g. $\boldsymbol{a}, \boldsymbol{b}$, etc.); and matrices, with bold uppercase letters (e.g. $\boldsymbol{A}, \boldsymbol{B}$, etc.). Furthermore, all vectors are column vectors unless otherwise stated, and $\mathbf{0}$ denotes a zero column vector of suitable dimensions. Transposition is denoted with the superscript ${ }^{\top}$, and the operators $<, \leq,=$, $\geq$ and $>$ denote element-wise relations of vectors.

\section{MODELING A NAVIGATION REACH}

In order to develop an MPC for inland waterways, a model that describes the dynamic behavior of the system is needed. The Saint-Venant nonlinear differential equations provide one of the most accurate mathematical representations of open-flow canals [11]. However, the lack of a general analytical solution and the sensitivity to errors in the parameters, among other reasons, make this set of equations not suitable for control purposes. For this reason, many simpler control-oriented models have been obtained by linearizing the Saint-Venant equations around an operating point and considering simplifications on the original equations. These simplified models must retain the representativeness of the main dynamics of the network as well as being as simple and flexible as possible while achieving the aims pursued [12].

The IDZ model presented in [13] is used for this purpose. Its main features are detailed, and its equivalent state-space representation is derived, which allows to take into account the effect of delayed inputs in a more convenient manner.

\section{A. The IDZ model}

The general IDZ input-output expression that links the discharges and the water depths at the boundaries of a reach is given by:

$$
\left[\begin{array}{l}
y(0, s) \\
y(L, s)
\end{array}\right]=\underbrace{\left[\begin{array}{ll}
p_{11}(s) & p_{12}(s) \\
p_{21}(s) & p_{22}(s)
\end{array}\right]}_{\mathbf{P}}\left[\begin{array}{l}
q(0, s) \\
q(L, s)
\end{array}\right],
$$

where 0 and $L$ are the abscissas for the initial and final ends of the canal; $y(0, s)$ and $y(L, s)$, the upstream and downstream water levels; $q(0, s)$ and $q(L, s)$, the upstream inflow and downstream outflow; and $p_{i j}(s)$, the different terms of the IDZ model, which consist of an integrator, a time delay and a zero:

$$
p_{i j}(s)=\frac{\alpha_{i j} \cdot s+1}{A_{i j} \cdot s} e^{-\tau_{i j} \cdot s}
$$

The physical interpretation of the parameters is as follows: $\alpha$ represents the inverse of the zero, which approximates through a constant gain the oscillatory phenomena that occurs in the high frequencies. $A$ is the integrator gain and accounts for the change in volume according to the variation of the water level. Finally, $\tau$ represents the propagation time delay, i.e. the required time for a wave to travel from its origin to the measurement points (therefore $\tau_{i i}=0$, assuming that the sensors are next to the actuators).

The parameters of the first equation of (1) are linked to the upstream water level, while those in the second equation are linked to the downstream water level. The authors in [13] modify the notation of the parameters based on this fact, which is followed in this work: $A_{11}=A_{12}=A_{u}, A_{21}=A_{22}$ $=A_{d}, \tau_{12}=\tau_{u}$ and $\tau_{21}=\tau_{d}$.

Note that the complete model is considered, which allows to take into account the backwater effect in the upstream water level. Indeed, it is common practice to control only the downstream water level [13].

\section{B. Equivalent state-space representation with delayed inputs}

In order to ensure the correct coordination between actual and past input values, an equivalent formulation is derived.

Equation (1) can be re-written as shown in (3). The following notation modifications are introduced for convenience: $y_{1}(s)=y(0, s), y_{2}(s)=y(L, s), q_{1}(s)=q(0, s)$ and $q_{2}(s)=q(L, s)$.

$$
\begin{aligned}
& y_{1}(s)=p_{11}(s) q_{1}(s)+p_{12}(s) q_{2}(s) \\
& y_{2}(s)=p_{21}(s) q_{1}(s)+p_{22}(s) q_{2}(s)
\end{aligned}
$$

Substituting (2) in (3) yields:

$$
\begin{aligned}
& y_{1}(s)=\frac{\alpha_{11} s+1}{A_{u} s} q_{1}(s)+\frac{\alpha_{12} s+1}{A_{u} s} e^{-\tau_{u} s} q_{2}(s) \\
& y_{2}(s)=\frac{\alpha_{21} s+1}{A_{d} s} e^{-\tau_{d} s} q_{1}(s)+\frac{\alpha_{22} s+1}{A_{d} s} q_{2}(s)
\end{aligned}
$$

As mentioned before, the discharges have a delayed effect on the off-diagonal entries of $\mathbf{P}$, i.e. the cases in which a sensor is not immediately next to an actuator.

To obtain the equivalent discrete state-space formulation of the IDZ model, (4) is discretized with a sampling time $T_{s}$. The final formulation reads as follows: 


$$
\begin{aligned}
\boldsymbol{x}_{k+1} & =\left[\begin{array}{cc}
1 & 0 \\
0 & 1
\end{array}\right] \boldsymbol{x}_{k}+\left[\begin{array}{cc}
T_{s} & 0 \\
0 & T_{s}
\end{array}\right] \boldsymbol{u}_{k}+\left[\begin{array}{cc}
0 & T_{s} \\
T_{s} & 0
\end{array}\right] \boldsymbol{u}_{k-n} \\
\boldsymbol{y}_{k} & =\left[\begin{array}{cc}
\frac{1}{A_{u}} & 0 \\
0 & \frac{1}{A_{d}}
\end{array}\right] \boldsymbol{x}_{k}+\left[\begin{array}{cc}
\frac{\alpha_{11}}{A_{u}} & 0 \\
0 & \frac{\alpha_{22}}{A_{d}}
\end{array}\right] \boldsymbol{u}_{k}+\left[\begin{array}{cc}
0 & \frac{\alpha_{12}}{A_{u}} \\
\frac{\alpha_{21}}{A_{d}} & 0
\end{array}\right] \boldsymbol{u}_{k-n}
\end{aligned}
$$

with $k$ the discrete-time instant and $\boldsymbol{u}_{k-n}$ the input vector delayed $n$ samples $\left(n=\left\lceil\tau / T_{s}\right\rceil\right.$, with $\lceil\cdot\rceil$ the ceiling function). Remark: in practice, the numerical values of $\tau_{d}$ and $\tau_{u}$ are almost the same, which leads to a single value of $n$.

Equation (5) regards the case of a reach with two controlled inputs and two outputs, and no uncontrolled discharges. The general formulation of a system with $n_{x}$ states, $n_{u}$ inputs and $n_{y}$ outputs, and with demands $\boldsymbol{d}_{k}$, is:

$$
\begin{aligned}
\boldsymbol{x}_{k+1} & =\boldsymbol{A} \boldsymbol{x}_{k}+\boldsymbol{B}_{u} \boldsymbol{u}_{k}+\boldsymbol{B}_{u-n} \boldsymbol{u}_{k-n}+\boldsymbol{B}_{d} \boldsymbol{d}_{k}+\boldsymbol{B}_{d-n} \boldsymbol{d}_{k-n} \\
\boldsymbol{y}_{k} & =\boldsymbol{C} \boldsymbol{x}_{k}+\boldsymbol{D}_{u} \boldsymbol{u}_{k}+\boldsymbol{D}_{u-n} \boldsymbol{u}_{k-n}+\boldsymbol{D}_{d} \boldsymbol{d}_{k}+\boldsymbol{D}_{d-n} \boldsymbol{d}_{k-n} \\
\mathbf{0} & =\boldsymbol{E}_{u} \boldsymbol{u}_{k}+\boldsymbol{E}_{d} \boldsymbol{d}_{k}
\end{aligned}
$$

with $\boldsymbol{x}_{k} \in \mathbb{R}^{n_{x}}, \boldsymbol{u}_{k} \in \mathbb{R}^{n_{u}}, \boldsymbol{y}_{k} \in \mathbb{R}^{n_{y}}$, and $\boldsymbol{A}, \boldsymbol{B}_{u}, \boldsymbol{B}_{u-n}, \boldsymbol{B}_{d}$, $\boldsymbol{B}_{d-n}, \boldsymbol{C}, \boldsymbol{D}_{u}, \boldsymbol{D}_{u-n}, \boldsymbol{D}_{d}, \boldsymbol{D}_{d-n}, \boldsymbol{E}_{u}$ and $\boldsymbol{E}_{d}$ are time-invariant matrices of suitable dimensions. In addition, $\boldsymbol{d}_{k} \in \mathbb{R}^{n_{d}}$ are the demands of the system, which act as additive disturbances. Since they correspond to uncontrolled inputs, they also have a delayed effect. More details will be given in Section III.C.

Equation (6a) is the state equation, (6b) is the output equation and (6c) describes the static relations (mass balances at the junction nodes) in the network. The latter can be formulated as a constraint, as it is now, or it can be incorporated into (6a) as shown in [14].

\section{PROBLEM FORMULATION}

Inland waterways are complex multivariable systems. MPC offers several important advantages when dealing with this type of systems [15]:

- The model captures the dynamic and static interactions between input, output and disturbance variables.

- The physical constraints on inputs and outputs can be handled systematically.

- It allows to take into account multiple objectives.

- It is particularly suitable for those systems for which the disturbances can be forecasted.

The main principle of MPC techniques resides in calculating a control sequence so that the predicted response moves to the setpoint in an optimal manner without violating the constraints. Therefore, in order to build the MPC problem, it is necessary to define the constraints and the operational goals that must be taken into account.

\section{A. System constraints}

The physical nature of the variables involved as well as some elements in the waterways constrain the system functioning. While these constraints should not be violated, they might be relaxed in certain occasions.

- Actuators: the MPC controller dictates the setpoints to the local controllers, which must ensure the correct flows through the actuators (e.g. gates, pumps and valves). These elements have lower and upper limits, regarded as constraints in the problem formulation:

$$
\underline{\boldsymbol{u}}^{m} \leq \boldsymbol{u}_{k}^{m} \leq \overline{\boldsymbol{u}}^{m}, m=1, \ldots, N_{m}
$$

where $\underline{\boldsymbol{u}}^{m}$ and $\overline{\boldsymbol{u}}^{m}$ are the minimum and the maximum flows that the $m$-th actuator can provide, and $N_{m}$ is the total number of actuators in the system.

- Nodes: inland waterways are characterized by streams that branch off from the main stream and flow away (distributaries) and, at some point, flow into larger streams or lakes (tributaries). Nodes represent the network points in which these splittings and mergings take place. Thus, they are regarded as mass balance relations modeled as equality constraints given by:

$$
\mathbf{0}=\boldsymbol{E}_{u} \boldsymbol{u}_{k}+\boldsymbol{E}_{d} \boldsymbol{d}_{k}
$$

In the case of natural mergings and splittings,

$$
y_{k}^{i_{l}}=y_{k}^{o_{l}}, l=1, \ldots, N_{l}
$$

with $i_{l}$ and $o_{l}$ the set of inflows and outflows at node $l$, respectively, and $N_{l}$ the total number of nodes in the system. This additional constraint imposes that the water levels at node $l$ for all the reaches with inflows and outflows at node $l$ must be exactly the same.

- Navigability condition: as mentioned before, it is crucial to maintain the water levels within the predefined navigation interval delimited by the Low and the High Navigation Levels (LNL and HNL, respectively) to ensure the navigability condition. These values are defined around the setpoint known as the Normal Navigation Level (NNL). Under certain circumstances, this constraint might be relaxed. To do so, a relaxation parameter $\boldsymbol{\alpha}_{k}$ is considered in the constraint and a quadratic penalty on this parameter is included in the objective function.

Thus, the navigability condition is formulated as:

$$
\underline{\boldsymbol{y}}_{r}-\boldsymbol{\alpha}_{k} \leq \boldsymbol{y}_{k} \leq \overline{\boldsymbol{y}}_{r}+\boldsymbol{\alpha}_{k}
$$

with $\underline{\boldsymbol{y}}_{r}$ and $\overline{\boldsymbol{y}}_{r}$ the lower (LNL) and upper (HNL) bounds of the NNL values, respectively.

\section{B. Operational goals and multi-objective function}

The set of control signals to be applied to the system is computed by optimizing a certain criterion (objective function), usually defined as the weighted sum of several terms, each of them accounting for an operational goal. Many different control objectives can be formulated depending on the operational goals that are to be fulfilled. In this work, the following goals are regarded:

- Maintaining the water levels close to the setpoints: this is the most important objective to be fulfilled. The mathematical formulation of this objective reads as:

$$
J_{k}^{1}=\left(\boldsymbol{y}_{k}-\boldsymbol{y}_{r}\right)^{\top}\left(\boldsymbol{y}_{k}-\boldsymbol{y}_{r}\right),
$$

with $\boldsymbol{y}_{r}$ the vector of NNL values.

- Cost reduction: the economic costs derived from the operation of the available controlled equipment (e.g. gates, pumps and valves) are reflected in this term. This 
can be mathematically formulated as:

$$
J_{k}^{2}=\boldsymbol{\gamma} \boldsymbol{u}_{k}^{\top} \boldsymbol{u}_{k}
$$

with $\boldsymbol{\gamma}$ the vector of known costs associated to the equipment operation.

- Smoothness of the control signal: in order to avoid damage to the equipment and increase its lifespan, a frequent approach to this issue consists in penalizing the control signal variation between consecutive time instants as follows:

$$
J_{k}^{3}=\Delta \boldsymbol{u}_{k}^{\top} \Delta \boldsymbol{u}_{k}
$$

with $\Delta \boldsymbol{u}_{k}=\boldsymbol{u}_{k}-\boldsymbol{u}_{k-1}$.

- Penalty in the relaxation parameter: the $\boldsymbol{\alpha}_{k}$ term introduced in (10) is penalized to ensure that the water levels are outside the navigation interval as little as possible:

$$
J_{k}^{4}=\boldsymbol{\alpha}_{k}^{\top} \boldsymbol{\alpha}_{k}
$$

The multi-objective function $J$ that gathers the previous control objectives can be described by

$$
J=\sum_{k=1}^{H_{p}} \sum_{j=1}^{4} \beta^{j} J_{k}^{j},
$$

where $H_{p}$ is the prediction horizon and $\beta^{j}$ are the weights of the $j$-th objective, selected based on a method used in the Pareto front calculation in a multi-objective optimization [16]. The main idea resides in computing a normalized objective space and finding the Pareto front point that minimizes the Euclidean distance to the management point.

\section{Control design}

The control-oriented model, the system constraints and the multi-objective function are gathered to build the MPC problem. The set of future control signals to be applied to the system is computed by optimizing the objective function (15) over the prediction horizon. At each time instant, a control signal sequence is computed over the considered control horizon $H_{u}$. However, only the first component of this sequence is applied to the system, and the rest are discarded.

The solution of the control problem is given by

$$
\min \sum_{k=1}^{H_{p}} \sum_{j=1}^{4} \beta^{j} J_{k}^{j}
$$

subject to:

$$
\begin{aligned}
& \begin{aligned}
\boldsymbol{x}_{k+i+1 \mid k}= & \boldsymbol{A} \boldsymbol{x}_{k+i \mid k}+\boldsymbol{B}_{u} \boldsymbol{u}_{k+i \mid k}+\boldsymbol{B}_{u-n} \boldsymbol{u}_{k+i-n \mid k} \\
& \quad+\boldsymbol{B}_{d} \boldsymbol{d}_{k+i \mid k}+\boldsymbol{B}_{d-n} \boldsymbol{d}_{k+i-n \mid k} \\
\boldsymbol{y}_{k+i \mid k}= & \boldsymbol{C} \boldsymbol{x}_{k+i \mid k}+\boldsymbol{D}_{u} \boldsymbol{u}_{k+i \mid k}+\boldsymbol{D}_{u-n} \boldsymbol{u}_{k+i-n \mid k} \\
& +\boldsymbol{D}_{d} \boldsymbol{d}_{k+i \mid k}+\boldsymbol{D}_{d-n} \boldsymbol{d}_{k+i-n \mid k}
\end{aligned} \\
& \begin{aligned}
\mathbf{0}=\boldsymbol{E}_{u} \boldsymbol{u}_{k+i \mid k}+\boldsymbol{E}_{d} \boldsymbol{d}_{k+i \mid k} \\
\underline{\boldsymbol{u}}^{m} \leq \boldsymbol{u}_{k}^{m} \leq \overline{\boldsymbol{u}}^{m}
\end{aligned} \\
& \underline{y}_{k}^{i_{l}}=y_{k}^{o_{l}} \\
& \underline{\boldsymbol{y}}_{r}-\boldsymbol{\alpha}_{k} \leq \boldsymbol{y}_{k} \leq \overline{\boldsymbol{y}}_{r}+\boldsymbol{\alpha}_{k} \\
& \boldsymbol{\alpha}_{k} \geq \mathbf{0}
\end{aligned}
$$

with $i$ the time instant along the prediction horizon, $k$ the current time instant and $k+i \mid k$ the instant $k+i$ given $k$. Equations (16b)-(16d) correspond to the model described by (5), while (16e)-(16h) are the constraints given in (7)-(10).

The demands $\boldsymbol{d}_{k}$ refer to the request of lock operations by the boat masters. Inland waterways are generally used for freight transport. Boats navigate along the system until their final destination, probably along several reaches in their way. Reaches are connected to one another by means of locks, which grant access to enclosures used to enable boats overcome the difference in elevation between two reaches.

Operating a lock implies that a rather large volume of water is discharged into the destination reach (along with the boat), which will disturb its equilibrium water level. However, a common waterways management policy dictates that, when a boat passes through a lock, its manager informs the manager of the next lock so that the arrival time of the boat can be anticipated. This information is obtained by taking into account the distance between the two locks and the average speed of the boat, which results in a close approximation. Therefore, the lock operation timeseries model can be elaborated in advance.

\section{CASE STUDY}

The result of applying the MPC given by the solution of (16) to the system is given in this section. First, the considered system is presented. Then, the problem formulation is particularized for this system. Finally, the performance of the controller is shown and the results are discussed.

\section{A. Description of the system}

A reach with a distributary branching off from the main stream is considered in this work. This case study is inspired by the large number of existing examples with this topology, and serves to illustrate the modeling and control steps that must be followed when dealing with multi-reach systems. A schematic overview is depicted in Fig. 1. The system is composed of three identical reaches, whose geometrical and physical parameters are summarized in Table I. $L$ is the length of the reaches, $w_{r}$ is the bottom width, $m_{r}$ is the side slope of the reach ( $m_{r}=0$ for a rectangular cross section), $s_{b}$ is the bottom slope ( $s_{b}=0$ for a flat reach), $n_{r}$ is the Manning roughness coefficient and $Q_{s}$ is the operating point considered in the linearization of the Saint-Venant equations.

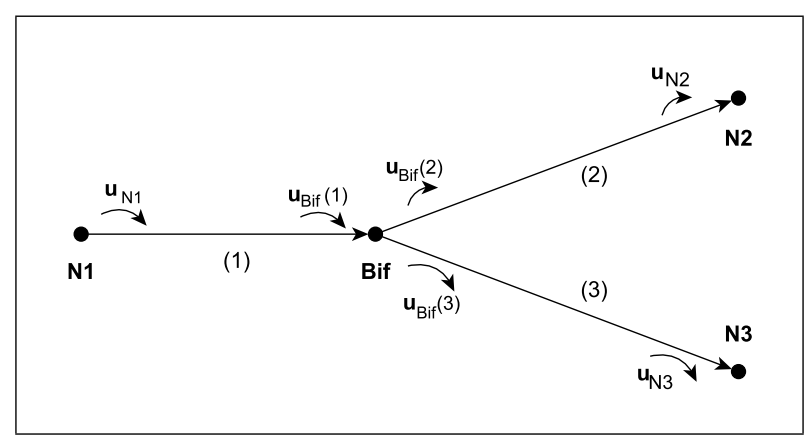

Fig. 1: Schematic diagram of the case study 
TABLE I: Parameters of the reaches

\begin{tabular}{cccccc}
\hline \hline$L[m]$ & $w_{r}[m]$ & $m_{r}[m / m]$ & $s_{b}[m / m]$ & $n_{r}\left[s / m^{1 / 3}\right]$ & $Q_{s}\left[m^{3} / s\right]$ \\
\hline 27000 & 50 & 0 & 0 & 0.035 & 0.6 \\
\hline \hline
\end{tabular}

$N 1, N 2$ and $N 3$ are the bounding nodes of the system. In each of these nodes, there is a controlled gate that allows to dispatch water to fulfill the control objectives, as well as a lock that enables boats entering and leaving the system. Bif stands for bifurcation, where there is only a gate that regulates the three flows (see Fig. 1). The existence of this gate overrides the condition imposed by (16f) as the bifurcation is not of natural type.

The navigability condition is guaranteed if the water levels are kept in the interval $3.8 \pm 0.10 \mathrm{~m}$. This objective is disturbed by the lock operations that take place each time that a boat needs to enter or leave the system. A lock operation in $N 1$ entails $18000 \mathrm{~m}^{3}$; in $N 2,-6000 \mathrm{~m}^{3}$; and in $N 3,-12000$ $\mathrm{m}^{3}$ (all of them last $20 \mathrm{~min}$ ). Remark: when a lock operation is performed, a certain amount of water is withdrawn from the upstream reach and released into the downstream reach. Thus, a lock operation at $N 1$ implies that the system is filled, whereas lock operations at $N 2$ and $N 3$ empty the system. This explanation accounts for the sign criterion adopted.

\section{B. Experimental design}

This system can be generally represented by the formulation given in (6). First, it is necessary to compute the IDZ parameters for each reach as specified in [13] and obtain the state-space representation by means of (5). A sampling time $T s=20 \mathrm{~min}$ is considered. The system matrices are built by stacking these local matrices. In particular, $\boldsymbol{B}_{d}, \boldsymbol{E}_{u}$ and $\boldsymbol{E}_{d}$ are obtained by analyzing how the input variables and the disturbances are linked and their effect on the other variables. As the system model is built as an aggregation of partial models, its dimensions are twice the number of reaches that make up the system. Due to lack of space, the final system matrices are not given in this work.

The node Bif is considered to obtain the mass balance relations. The physical upper and lower bounds of the actuators (operational design limits) are taken into account, which are $\pm 60 \mathrm{~m}^{3} / \mathrm{s}$ (the water flow can be injected in both directions), and the $\underline{y}_{r}, \boldsymbol{y}_{r}$ and $\overline{\boldsymbol{y}}_{r}$ values specified in Section IV.A are used. Finally, the weighting parameters $\beta^{j}$ are selected according to the relative importance of the control objectives.

A 24-hour navigation period is simulated to show the performance of the proposed approach and is depicted in Fig. 2. This scenario has been designed by taking into account the features that characterize real inland waterways:

- Two different periods are considered in a 24-hour interval, a navigation period and a stoppage period. The navigation period is considered to start at 6 a.m. and lasts for fourteen hours, until $8 \mathrm{p} . \mathrm{m}$. The navigation is then interrupted for ten hours, until the next day at 6 a.m.
- The considered lock operation magnitudes as well as the navigation demand (number of lock operations per day) correspond to realistic values, typical of this type of systems.

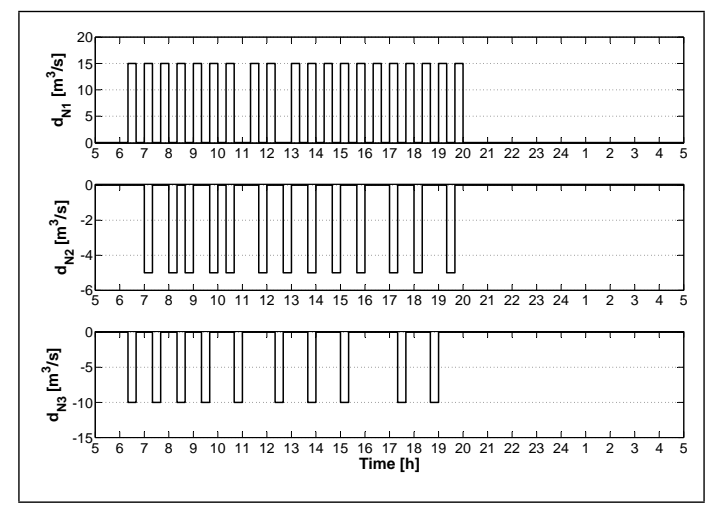

Fig. 2: Lock operation time-series model

\section{Results}

The following results are obtained after simulating the described scenario. The controlled discharges computed by the predictive controller are shown in Fig. 3 while the predicted water levels are depicted in Fig. 4. Since there exists a proportionality between output and state variables as shown in (5), the state variables are not presented. Note also that there are three variables for the bifurcation node, one for each reach. The distinction is made by adding the reach number after the variable as shown in Fig. 1.

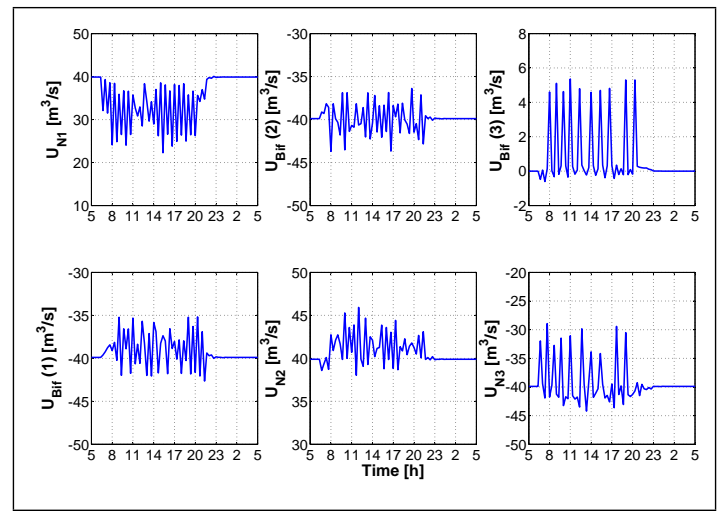

Fig. 3: Computed controlled discharges

The simulation starts at 5 a.m., one hour before the navigation period. The system is disturbed during the navigation period, and the water levels return to their equilibrium values after the navigation is interrupted. Fig. 4 shows that the MPC is able to keep the water levels inside the navigation interval in the presence of disturbances. However, it is worth noting that the equilibrium water levels once the navigation period stops do not correspond exactly to the NNL values. The mass transport during the simulation as well as the final mass balance accounts for this difference.

On the other hand, the set of control actions is kept within the equipment design range. Nevertheless, it must be 


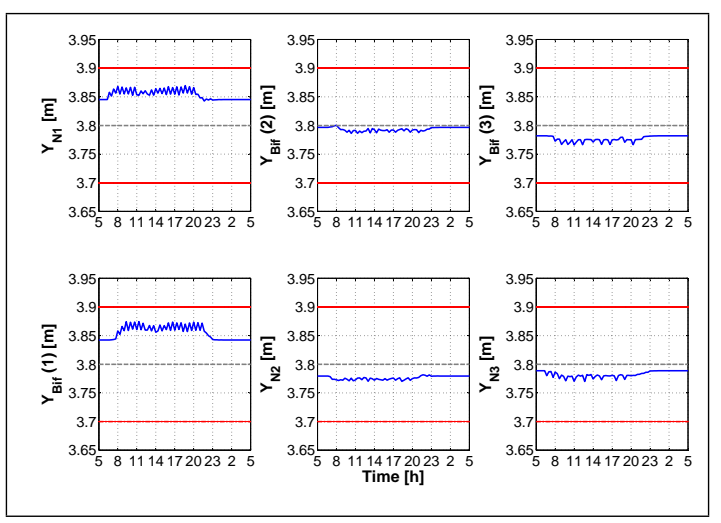

Fig. 4: Water levels $\boldsymbol{y}_{k}$ (blue solid lines), navigation intervals (red dash-dot lines) and setpoints $\boldsymbol{y}_{r}$ (gray dashed lines)

recalled that one of the operational goals was to guarantee a smooth control signal by minimizing the difference between consecutive control actions. Even if the signals depicted in Fig. 3 do not exhibit the smoothest behavior, there are no large differences compared to the operational range. Moreover, the control action values are far from the limits, which should result in a long lifespan of the equipment.

To quantify the MPC performance, consider the tracking indices

$$
T E[\%]=100 *\left[1-\frac{1}{H_{p}} \sqrt{\sum_{k=1}^{H_{p}}\left(\frac{\boldsymbol{y}_{k}-\boldsymbol{y}_{r}}{\frac{1}{2}\left(\overline{\boldsymbol{y}}_{r}-\underline{y}_{r}\right)}\right)^{2}}\right]
$$

defined as the relative error between the predicted levels $\boldsymbol{y}_{k}$ and the setpoints $\boldsymbol{y}_{r}$, and $\frac{1}{2}\left(\overline{\boldsymbol{y}}_{r}-\underline{\boldsymbol{y}}_{r}\right)$ is the maximum allowed variation from $\boldsymbol{y}_{r}$. The numerical values are summarized in Table II ( $T E=100 \%$ corresponds to the perfect tracking). It can be stated that the control approach provides satisfactory results and the tracking performance is guaranteed.

TABLE II: Tracking performances

\begin{tabular}{ccccccc}
\hline \hline & N1 & Bif (1) & Bif (2) & N2 & Bif (3) & N3 \\
\hline TE[\%] & 93.64 & 93.37 & 99.14 & 97.21 & 97.25 & 97.89 \\
\hline \hline
\end{tabular}

\section{CONCLUSIONS}

An MPC approach for inland waterways was presented in this paper. To do so, the existing IDZ model was adapted to solve the two main issues that are dealt with: the backwater effect, which appears in open-flow water systems with negligible bottom slope, and the correct management of the manipulated inputs when the system is characterized by large time delays, which have an immediate and a delayed effect in the network. To illustrate the approach and test its performance, a realistic case study was considered. The operational goals and the constraints were formulated, and the model was adapted to the particular topology of the case.

In the light of the results, the system exhibits a satisfactory performance. Nevertheless, this work constitutes a first step towards the final solution. Indeed, this approach is designed to be tested in a part of the inland waterways in the north of France. Several aspects such as uncontrolled bifurcations and different time delays in the network must be considered. Distributed control techniques will be probably used to find an efficient solution. Finally, the effect of possible sensor and actuator faults in the system will be considered, and therefore fault-tolerant control techniques will be developed.

\section{ACKNOWLEDGMENTS}

This work has been partially funded by the Spanish State Research Agency (AEI) and the European Regional Development Fund (ERFD) through the projects DEOCS (ref. MINECO DPI2016-76493) and SCAV (ref. MINECO DPI2017-88403-R). This work has also been partially funded by AGAUR of Generalitat de Catalunya through the Advanced Control Systems (SAC) group grant (2017 SGR 482).

\section{REFERENCES}

[1] S. Mihic, M. Golusin, and M. Mihajlovic, "Policy and promotion of sustainable inland waterway transport in Europe - Danube river," Renewable and Sustainable Energy Rev., vol. 15, pp. 1801-1809, 1993.

[2] Intergovernmental Panel on Climate Change, Climate Change 2014 Impacts, Adaptation and Vulnerability: Regional Aspects. Cambridge University Press, 2014.

[3] P. O. Malaterre and J. Rodellar, "Multivariable predictive control of irrigation canals. design and evaluation on a 2-pool model," in Int. Workshop on Regulation of Irrigation Canals, 1997, pp. 230-238.

[4] A. Álvarez, M. A. Ridao, D. R. Ramirez, and L. Sánchez, "Constrained predictive control of an irrigation canal," Journal of Irrigation and Drainage Engineering, vol. 139, no. 10, pp. 841-854, 2013.

[5] O. Begovich, V. M. Ruiz, G. Besançon, C. I. Aldana, and D. Georges, "Predictive control with constraints of a multi-pool irrigation canal prototype," Latin American App. Research, vol. 37, no. 3, pp. 177$185,2007$.

[6] P. J. Van Overloop, R. R. Negenborn, B. De Schutter, and N. C. Van De Giesen, "Predictive control for national water flow optimization in The Netherlands," Intelligent Infrastructures, vol. 42, pp. 439-461, 2010.

[7] A. Zafra-Cabeza, J. M. Maestre, M. A. Ridao, E. F. Camacho, and L. Sánchez, "A hierarchical distributed model predictive control approach to irrigation canals: a risk mitigation perspective," Journal of Process Control, vol. 21, no. 5, pp. 787-799, 2011.

[8] V. Puig, C. Ocampo-Martínez, and R. R. Negenborn, "Model predictive control for combined water supply and navigability/sustainability in river systems," in Transport of Water versus Transport over Water. Springer, 2015, pp. 13-33.

[9] K. Horváth, L. Rajaoarisoa, E. Duviella, J. Blesa, M. Petreczky, and K. Chuquet, "Enhancing inland navigation by model predictive control of water levels: the Cuinchy-Fontinettes case," in Transport of Water versus Transport over Water. Springer, 2015, pp. 211-234.

[10] P. Segovia, L. Rajaoarisoa, F. Nejjari, V. Puig, and E. Duviella, "Decentralized control of inland navigation networks with distributaries: application to navigation canals in the north of France," in 2017 American Control Conference (ACC), May 2017, pp. 3341-3346.

[11] V. T. Chow, Open-channel hydraulics. New York: McGraw-Hill Book Co. Inc, 1959.

[12] C. Ocampo-Martínez, V. Puig, G. Cembrano, and J. Quevedo, "Application of predictive control strategies to the management of complex networks in the urban water cycle," IEEE Control Systems Magazine, vol. 33 , no. 1 , pp. 15-41, 2013.

[13] X. Litrico and V. Fromion, Modeling and Control of Hydrosystems. Springer, 2009.

[14] J. M. Grosso, C. Ocampo-Martínez, V. Puig, and B. Joseph, "Chanceconstrained model predictive control for drinking water networks," Journal of Process Control, vol. 24, no. 5, pp. 504-516, 2014.

[15] E. F. Camacho and C. Bordons, Model Predictive Control. London: Springer, 1998.

[16] R. Toro, C. Ocampo-Martínez, F. Logist, J. Van Impe, and V. Puig, "Tuning of predictive controllers for drinking water networked systems," IFAC Proceedings Volumes, vol. 44, no. 1, pp. 14 507-14512, 2011. 